\title{
ЗАДАЧА С ИНТЕГРАЛЬНЫМ УСЛОВИЕМ ДЛЯ ОДНОМЕРНОГО ГИПЕРБОЛИЧЕСКОГО УРАВНЕНИЯ
}

\begin{abstract}
В статье рассматривается нелокальная задача с интегральным условием для одномерного гиперболического уравнения, возникающая при исследовании колебаний стержня. Получены условия на входные данные, обеспечивающие однозначную разрешимость поставленной задачи, проведено доказательство существования и единственности решения задачи.
\end{abstract}

Ключевые слова: гиперболическое уравнение, нелокальная задача, интегральные условия, единственность решения, разрешимость задачи.

Цитирование. Богатов А.В. Задача с интегральным условием для одномерного гиперболического уравнения // Вестник Самарского университета. Естественнонаучная серия. 2018. Т. 24. № 4. С. 7-12. DOI: http://doi.org/10.18287/2541-7525-2018-24-4-7-12.

\section{Введение}

В статье изучается задача для одномерного гиперболического уравнения с нелокальным интегральным условием первого рода.

Задачи с нелокальными условиями представляют собой одно из динамично развивающихся направлений современной теории дифференциальных уравнений. Нелокальными краевыми задачами принято называть задачи, в которых задаются условия, связывающие значения искомого решения или его производных в различных точках границы и каких-либо внутренних точках. Отдельного внимания заслуживает класс задач с интегральными условиями, которые являются обобщением локальных и дискретных нелокальных условий. Нелокальные интегральные условия возникают при исследовании различных физических явлений в случае, когда граница области протекания процесса недоступна для непосредственного измерения. Нелокальные задачи также имеют большое практическое значение при решении задач механики твердого тела, а именно, они позволяют построить математическую модель процесса неразрушающего контроля и, как следствие, управлять напряженно-деформированным состоянием.

Уравнение колебаний струны является простейшим уравнением гиперболического типа и используется для описания линейных волновых процессов различной физической природы. Например, это уравнение описывает малые поперечные колебания струны, продольные колебания тонкого стержня, применяется при рассмотрении широкого круга волновых процессов акустики, гидродинамики, электродинамики. Исследования задач с нелокальными условиями показали, что многие классические методы доказательства разрешимости начально-краевых задач не применимы в случае нелокальных задач или требуют значительной модификации. Попытка применить метод разделения переменных для исследования разрешимости задач с интегральными условиями чаще всего приводит к неортогональной и неполной системе собственных функций, что в свою очередь делает метод разделения переменных неприменимым. Серьезной причиной неприменимости классических методов для исследования нелокальных задач является тот факт, что область определения оператора, порождаемого нелокальной задачей, как правило, не является плотной в $L_{2}$. Поэтому разработка методов исследования разрешимости нелокальных задач остается актуальной. Несмотря на то, что на сегодняшний день разработан не один метод решения нелокальных задач, мы воспользовались одним из самых универсальных - методом вспомогательных задач. Суть метода вспомогательных задач в следующем: сначала решается задача с классическими (но неизвестными) граничными условиями, а затем производится попытка поиска граничных условий как

\footnotetext{
${ }^{1}$ (C) Богатов А.В., 2018

Богатов Андрей Владимирович (andrebogato@mail.ru), кафедра дифференциальных уравнений и теории управления, Самарский национальный исследовательский университет имени академика С.П. Королева, 443011, Российская Федерация, г. Самара, Московское шоссе, 34.
} 
решения операторного уравнения, полученного в результате применения нелокального условия к решению вспомогательной задачи. Именно этим методом, хотя он тогда еще не имел названия, доказана разрешимость нелокальной задачи с интегральным условием для уравнения теплопроводности в статье Дж. Р. Кэннона [1]. Нелокальные задачи для дифференциальных уравнений рассматривались многими авторами. Отметим здесь статьи [2-4], а также монографию [5] и обратим внимание на список литературы в них. Заметим, что задачи с интегральными условиями часто можно трактовать как задачи управления. Задачам управления для уравнения колебания струны посвящен ряд статей В.А. Ильина $[6 ; 7]$.

Колебательные процессы могут возникать в абсолютно любой механической системе. Причины появления в зависимости от типов источников делятся на два типа: внутренние и внешние источники. Многие механизмы, как правило, представляют собой стержни переменного сечения. Например, балка моста автомобиля: к центру идет расширение диаметра стержня за счет отверстия для слива масла, крышки картера, рессорной подушки, и т.д., в авиационных двигателях - лопатки турбины, в устройствах обработки материалов - волноводы [8-10]. То есть можно сделать вывод, что в различных областях техники решаются задачи, связанные с колебаниями стержней. Для обеспечения долгой и бесперебойной работы современной техники необходимо проводить регулярные диагностические процедуры для оценки состояния и возможности дальнейшей эксплуатации оборудования. Но не всегда представляется возможным провести практическую диагностику оборудования, поэтому анализ результатов диагностики приходится проводить на основе косвенной информации о процессе колебаний (чаще всего - некоторое среднее значение). Если переходить к математической модели, то эта информация как правило поступает в виде интеграла от искомого решения.

Как было отмечено ранее, причины и следствия протекания колебательных процессов могут быть различными, то есть возникает необходимость теоретического изучения колебаний с целью определения допустимых областей изменения параметров, влияющих на протекание процесса. Исследованию математических моделей колебательных процессов посвящено большое количество статей, отметим здесь некоторые из них: [11-14].

В нашей статье рассматривается задача для одномерного гиперболического уравнения, возникающая при исследовании колебаний стержня.

\section{1. Постановка задачи}

Рассмотрим следующую задачу: в области $Q_{T}=(0, l) \times(0, T)$ найти решение уравнения

$$
u_{t t}-a^{2} u_{x x}=f(x, t),
$$

удовлетворяющее начальным данным

$$
u(x, 0)=\phi(x), u_{x}(0, t)=0,
$$

и нелокальному условию

$$
\int_{0}^{l} K_{i}(x) u(x, t) d x=g(t) .
$$

Стоит отметить, что (1.3) - операторное уравнение первого рода, которое является некорректно поставленной задачей. Для ее решения необходимо установить ограничения на ядро и правую часть.

\section{2. Разрешимость задачи}

Теорема 2.1 Если $K_{i} \in C^{\prime}[0, l], \triangle=K_{1}(0) K_{2}(l)-K_{1}(l) K_{2}(0) \neq 0, f \in C^{\prime}\left(\bar{Q}_{T}\right)$, при всех $T \leqslant l$, то существует единственное решение $u \in C^{2}\left(Q_{T}\right) \cap C\left(\bar{Q}_{T}\right)$ задачи (1.1)-(1.3)

\section{Доказательство}

На данный момент известен не один метод решения задач с нелокальными условиями, но мы применим метод вспомогательных задач, поскольку он применим в рамках поставленной задачи и является одним из самых надежных методов.

В качестве вспомогательной задачи рассмотрим первую начально-краевую задачу с неоднородными краевыми условиями

$$
\begin{gathered}
u_{t t}-a^{2} u_{x x}=f(x, t), \\
u(0, t)=\mu_{1}(t), u(l, t)=\mu_{2}(t), \mu_{1}(t) \in W_{2}^{2}(0, T), \mu_{i}(t)=\mu_{i}^{\prime}(t), t \leqslant 0 .
\end{gathered}
$$


Будем искать ее решение в виде суммы $u(x, t)=u^{0}(x, t)+v(x, t)$, где $u^{0}(x, t)$ - решение задачи для однородного уравнения

$$
\left\{\begin{array}{r}
u_{t t}^{0}-a^{2} u_{x x}^{0}=0, \\
u^{0}(0, t)=\mu_{1}(t), \\
u^{0}(l, t)=\mu_{2}(t), \\
u^{0}(x, 0)=0 \\
u_{t}^{0}=0
\end{array}\right.
$$

а $v(x, t)$ - решение задачи

$$
\left\{\begin{array}{r}
v_{t t}-a^{2} v_{x x}=f(x, t) \\
v(0, t)=v(l, t)=0 \\
v(x, 0)=v_{t}(x, 0)=0
\end{array}\right.
$$

Для решения задачи $(2.2)$ воспользуемся приемом, предложенным в статье [2]. Пусть функции $\mu_{i} \in$ $W_{2}^{2}(0, T), \quad \mu_{i}(t)=0$ при $t \leqslant 0$.

Тогда решение задачи (2.2) можно представить следующим образом:

$$
u^{0}(x, t)=\mu_{1}(t-x)+\mu_{2}(t+x-l), T \leqslant l .
$$

Для решения задачи (2.3) применим классический метод разделения переменных, что приведет к представлению решения

$$
v(x, t)=\sum_{n=1}^{\infty} V_{n}(t) \sin \frac{\pi n x}{l}, V_{n}(t)=\frac{l}{\pi n a} \int_{0}^{t} f_{n}(\theta) \sin \frac{\pi n}{l}(t-\tau) d \tau .
$$

Тогда решение вспомогательной задачи (2.1) имеет вид

$$
u(x, t)=\mu_{1}(t-x)+\mu_{2}(t+x-l)+\sum_{n=1}^{\infty} V_{n}(t) \sin \frac{\pi n x}{l}
$$

Однако функции $\mu_{i}(t)$ нам не известны. Будем пытаться их найти, применив к (2.4) интегральные условия (2.1).

В результате мы приходим к системе уравнений

$$
\begin{aligned}
& \int_{0}^{l} K_{1}(x) \mu_{1}(t-x) d x+\int_{0}^{l} K_{1}(x) \mu_{2}(t+x-l) d x+\sum_{n=1}^{\infty} V_{n}(t) \int_{0}^{l} K_{1}(x) \sin \frac{\pi n x}{l}=g_{1}(t), \\
& \int_{0}^{l} K_{2}(x) \mu_{1}(t-x) d x+\int_{0}^{l} K_{2}(x) \mu_{2}(t+x-l) d x+\sum_{n=1}^{\infty} V_{n}(t) \int_{0}^{l} K_{2}(x) \sin \frac{\pi n x}{l}=g_{2}(t) .
\end{aligned}
$$

Теперь нам нужно выяснить, найдутся ли функции, удовлетворяющие этой системе интегральных уравнений. При положительном ответе на этот вопрос, формула (2.4) даст решение поставленной нелокальной задачи.

В (2.5) сделаем замену переменных, положив $\xi=t-x, \xi=x+t-l$ в первых двух интегралах каждого уравнения. Тогда, учитывая свойства функции $\mu_{i}(t)$ получим

$$
\begin{aligned}
& \int_{0}^{l} K_{1}(t-\xi) \mu_{1}(\xi) d \xi+\int_{0}^{l} K_{1}(\xi-t+l) \mu_{2}(\xi) d \xi+\sum_{n=1}^{\infty} V_{n}(t) \int_{0}^{l} K_{1}(x) \sin \frac{\pi n x}{l} d x=g_{1}(t), \\
& \int_{0}^{l} K_{2}(t-\xi) \mu_{1}(\xi) d \xi+\int_{0}^{l} K_{2}(\xi-t+l) \mu_{2}(\xi) d \xi+\sum_{n=1}^{\infty} V_{n}(t) \int_{0}^{l} K_{2}(x) \sin \frac{\pi n x}{l} d x=g_{2}(t) .
\end{aligned}
$$

Дифференцируя уравнения (2.6) по t, в силу условия теоремы, получим систему интегральных уравнений Вольтерра второго рода:

$$
K_{1}(0) \mu_{1}(t)+K_{1}(l) \mu_{2}(t)+\int_{0}^{t} K_{1}^{\prime}(t-\xi) \mu_{1}(\xi) d \xi-\int_{0}^{t} K_{1}^{\prime}(\xi-t+l) \mu_{2}(\xi) d \xi=\overline{g_{1}}(t)
$$


где

$$
K_{2}(0) \mu_{1}(t)+K_{2}(l) \mu_{2}(t)+\int_{0}^{t} K_{2}^{\prime}(t-\xi) \mu_{1}(\xi) d \xi-\int_{0}^{t} K_{2}^{\prime}(\xi-t+l) \mu_{2}(\xi) d \xi=\overline{g_{2}}(t)
$$

$$
\bar{g}_{i}(t)=g_{i}^{\prime}(t)-\sum_{n=1}^{\infty} V_{n}(t) \int_{0}^{l} K_{2}(x) \sin \frac{\pi n x}{l} d x .
$$

Так как по условию теоремы $\triangle=K_{1}(0) K_{2}(l)-K_{1}(l) K_{2}(0) \neq 0$, то $(2.7)$ можно разрешить относительно $\mu_{i}(t)$ :

$$
\begin{aligned}
& \mu_{1}(t)+\int_{0}^{t} \aleph_{11}(\xi, t) \mu_{1}(\xi) d \xi+\int_{0}^{t} \aleph_{12}(\xi, t) \mu_{2}(\xi) d \xi=g_{1}(t), \\
& \mu_{2}(t)+\int_{0}^{t} \aleph_{21}(\xi, t) \mu_{1}(\xi) d \xi+\int_{0}^{t} \aleph_{22}(\xi, t) \mu_{2}(\xi) d \xi=g_{1}(t),
\end{aligned}
$$

где

$$
\begin{gathered}
H_{11}(\xi, t)=\frac{K_{1}^{\prime}(t-\xi) K_{2}(l)-K_{2}^{\prime}(t-\xi) K_{1}(l)}{\triangle}, \\
H_{12}(\xi, t)=\frac{K_{2}^{\prime}(t-\xi) K_{1}(l)-K_{1}^{\prime}(t-\xi) K_{2}(l)}{\triangle}, \\
H_{21}(\xi, t)=\frac{K_{1}^{\prime}(t-\xi) K_{2}(l)-K_{2}^{\prime}(t-\xi) K_{1}(l)}{\triangle}, \\
H_{22}(\xi, t)=\frac{K_{1}(l) K_{2}^{\prime}(t-\xi)-K_{2}(l) K_{1}^{\prime}(t-\xi)}{\triangle}, \\
G_{1}(t)=\frac{\overline{g_{1}}(t) K_{2}(l)-\overline{g_{2}}(t) K_{1}(l)}{\triangle}, \\
G_{2}(t)=\frac{\overline{g_{1}}(t) K_{2}(l)-\overline{g_{2}}(t) K_{1}(l)}{\triangle} .
\end{gathered}
$$

В силу условий теоремы, нетрудно убедиться в том, что $H_{i j}(\xi, t)$ ограничены, $G_{i}(t)$ интегрируемы на $[0, \mathrm{~T}]$. Тогда система (2.8) однозначно разрешима [15].

Итак, функции $\mu_{i}(t)$ однозначно определены из системы интегральных уравнений, которая является следствием применения интегральных условий. Тогда решение вспомогательной задачи с найденными функциями $\mu_{i}(t)$ в качестве граничных условий представляет собой решение поставленной задачи.

\section{Литература}

[1] Cannon J.R. The solution of the heat equation subject to the specification of energy // Quart. Appl. Math. 1963. № 21. URL: https://www.jstor.org/stable/43635292.

[2] Пулькина Л.С. Краевые задачи для гиперболического уравнения с нелокальными условиями I и II рода // Изв. вузов. Сер.: Математика. 2012. № 4. С. 74-83. URL: http://mi.mathnet.ru/ivm8596.

[3] Гордезиани Д.Г., Авалишвили Г.А. Решения нелокальных задач для одномерных колебаний среды // Матем. моделир. 2000. № 1. С. 94-103. URL: http://mi.mathnet.ru/mm832.

[4] Avalishvili G., Avalishvili M., Gordeziani D. On integral nonlocal boundary problems for some partial differential equations // Bulletin of the Georgian National Academy of Sciences. 2011. 5(1). C. 31-37. URL: http://science.org.ge/old/moambe/5-1/31-37\%20Avalishvili.pdf.

[5] Пулькина Л.С. Задачи с неклассическими условиями для гиперболических уравнений. Самара: Изд-во Самарский университет, 2012.

[6] Ильин В.А., Тихомиров В.В. Волновое уравнение с граничным управлением на двух концах и задача о полном успокоении колебательного процесса // Дифференц. уравнения. 1999. Т. 35. №5. С. $692-304$. URL: http://mi.mathnet.ru/de9920.

[7] Ильин В.А., Моисеев Е.И. О единственности решения смешанной задачи для волнового уравнения с нелокальными граничными условиями // Дифференц. уравнения. 2000. Т. 36. № 5. C. 656-661. DOI: https://doi.org/10.1007/BF02754231.

[8] Хазанов Х.С. Механические колебания систем с распределенными параметрами: учеб. пособие. Самара: Самар. Госуд. Аэрокосмич. УН-т, 2002. 80 с. 
[9] Вейц В.Л., Дондошанский В.К., Чиряев В.И. Вынужденные колебания в металлорежущих станках. М.; Л.: Машгиз, 1959. 288 с.

[10] Нерубай М.С., Штриков Б.Л., Калашников В.В. Ультразвуковая механическая обработка и сборка. Самара: Самарское книжное изд-во, 1995. 191 с.

[11] Бейлин А.Б., Пулькина Л.С. Задача о колебаниях стержня с неизвестным условием его закрепления на части границы // Вестник Самарского университета. Естественнонаучная серия. 2017. Т. 23. № 2. С. 7-14. DOI: http://dx.doi.org/10.18287/2541-7525-2017-23-2-7-14.

[12] Федотов И.А., Полянин А.Д., Шаталов М.Ю. Теория свободных и вынужденных колебаний твердого стержня, основанная на модели Рэлея // Докдады РАН. 2007. Т. 417. № 1. С. 56-61.

[13] Бейлин А.Б., Пулькина Л.С. Задача о продольных колебаниях стержня с динамическими граничными условиями // Вестник СамГУ. Естественнонаучная серия. 2014. №3(114). C. 9-19. URL: http://vestnikoldsamgu.ssau.ru/articles/3-2014-1.pdf.

[14] Бейлин А.Б. Задача о продольных колебаниях упруго закреплённого нагруженного стержня // Вестник Самарского гос. Тех. Ун-та. Сер.: Физ.мат. науки. 2016. Т. 20. №2. С. 249-258. DOI: https://doi.org/10.14498/vsgtu1474.

[15] Михлин С.Г. Линейные уравнения в частных производных. М.: Высшая школа, 1977. 241 с.

\section{References}

[1] Cannon J.R. The solution of the heat equation subject to the specification of energy. Quart. Appl. Math., 1963, no. 21. Available at: https://www.jstor.org/stable/43635292 [in Russian].

[2] Pulkina L.S. Kraevye zadachi dlya giperbolicheskogo uravneniya s nelokalnymi usloviyami I $i$ II roda [Boundary value problems for the hyperbolic equation with nonlocal conditions of the I and II kind]. Izv. vuzov. Ser.: Matematika [Russian Mathematics], 2012, no. 4, pp. 74-83. Available at: http://mi.mathnet.ru/ivm8596 [in Russian].

[3] Gordeziani D.G., Avalishvili G.A. Resheniya nelokalnykh zadach dlya odnomernykh kolebanii sredy [Solutions of nonlocal problems for one-dimensional oscillations]. Matem. modelir. [Mathematical Models and Computer Simulations], 2000, no. 1, pp. 94-103. Available at: http://mi.mathnet.ru/mm832 [in Russian].

[4] Avalishvili G., Avalishvili M., Gordeziani D. On integral nonlocal boundary problems for some partial differential equations. Bulletin of the Georgian National Academy of Sciences, 2011, no. 5(1), pp. 31-37. Available at: http://science.org.ge/old/moambe/5-1/31-37\%20Avalishvili.pdf [in English].

[5] Pulkina L.S. Zadachi s neklassicheskimi usloviyami dlya giperbolicheskikh uravnenii [Problems with non-classical conditions for hyperbolic equations]. Samara: Izd-vo Samarskii universitet, 2012 [in Russian].

[6] Ilyin V.A., Tikhomirov V.V. Volnovoe uravnenie s granichnym upravleniem na dvukh kontsakh $i$ zadacha o polnom uspokoenii kolebatelnogo protsessa [Wave equation with boundary control at two ends and the problem of complete calming of oscillatory process]. Differents. uravneniya [Differential Equations], 1999, Vol. 35, no. 5, pp. 697-708. Available at: http://mi.mathnet.ru/de9920 [in Russian].

[7] Ilyin V.A., Moiseev E.I. O edinstvennosti resheniya smeshannoi zadachi dlya volnovogo uravneniya s nelokalnymi granichnymi usloviyami [On the uniqueness of solution of a mixed problem for the wave equation with nonlocal boundary conditions]. Differents. uravneniya [Differential Equations], 2000, Vol. 36, no. 5, pp. 728-733. DOI: https://doi.org/10.1007/BF02754231 [in Russian].

[8] Khazanov Kh.S. Mekhanicheskie kolebaniya sistem s raspredelennymi parametrami: ucheb. posobie [Mechanical vibrations of systems with distributed parameters: textbook]. Samaara: Samar. Gosud. Aerokosmich. Un-t, 2002, 80 p. [in Russian].

[9] Veits V.L., Dondoshanskiy V.K., Chiryaev V.I. Vynuzhdennye kolebaniya v metallorezhushchikh stankakh [Forced oscillations in metal-cutting machines]. M.; L.: Mashgiz, 1959, 288 p. [in Russian].

[10] Nerubay M.S., Shtrikov B.L., Kalashnikov V.V. Ultrazvukovaya mekhanicheskaya obrabotka i sborka [Ultrasonic machining and assembly]. Samara: Samarskoe knizhnoe izd-vo, 1995, 191 p. [in Russian].

[11] Beylin A.B., Pulkina L.S. [Zadacha o kolebaniyakh sterzhnya s neizvestnym usloviem ego zakrepleniya na chasti granitsy] [The problem of oscillations of a rod with an unknown condition for its attachment to a part of the boundary]. Vestnik Samarskogo universiteta. Estestvennonauchnaya seriya [Vestnik of Samara University. Natural Science Series], 2017. Vol. 23, no. 2, pp. 7-14. DOI: http://dx.doi.org/10.18287/2541-7525-2017-23-2-7-14 [in Russian].

[12] Fedotov I.A., Polyanin A.D., Shatalov M.Yu. Teoriya svobodnykh $i$ vynuzhdennykh kolebanii tverdogo sterzhnya, osnovannaya na modeli Releya [Theory of free and forced oscillations of a solid rod based on Rayleigh model]. Dokdady RAN [Doklady Mathematics], 2007, Vol. 417, no. 1, pp. 56-61 [in Russian]. 
[13] Beylin A.B., Pulkina L.S. Zadacha o prodolnykh kolebaniyakh sterzhnya s dinamicheskimi granichnymi usloviyami [Problem of longitudinal vibrations of a rod with dynamic boundary conditions]. Vestnik SamGU. Estestvennonauchnaya seriya [Vestnik of Samara State University. Natural Science Series], 2014, no. 3(114), pp. 9-19. Available at: http://vestnikoldsamgu.ssau.ru/articles/3-2014-1.pdf [in Russian].

[14] Beylin A.B. Zadacha o prodolnykh kolebaniyakh uprugo zakreplennogo nagruzhennogo sterzhnya [Problem of longitudinal vibrations of an elastically fixed loaded rod]. Vestnik Samarskogo gos. Tekh. Un-ta. Ser.: Fiz.-mat. nauki [Vestnik of Samara State Technical University. Technical Sciences Series], 2016, Vol. 20, no. 2, pp. $249-258$. DOI: https://doi.org/10.14498/vsgtu1474 [in Russian].

[15] Mikhlin S.G. Lineinye uravneniya $v$ chastnykh proizvodnykh [Linear partial differential equations]. M.: Vysshaya shkola, 1977, 241 p. [in Russian].

\section{A.V. Bogatov ${ }^{2}$}

\section{PROBLEM WITH AN INTEGRAL CONDITION FOR ONE-DIMENSIONAL HYPERBOLIC EQUATION}

In this paper, we study a nonlocal problem with an integral condition for a one-dimensional hyperbolic equation arising in the study of vibrations of the rod. The conditions for the input data providing unambiguous solvability of the problem are obtained, the proof of the existence and uniqueness of the solution of the problem is carried out.

Key words: hyperbolic equation, nonlocal proiblem, integral conditions, uniqueness of the solution, solvability of the problem.

Citation. Bogatov A.V. Zadacha $s$ integralnym usloviem dlya odnomernogo giperbolicheskogo uravneniya [Problem with an integral condition for one-dimensional hyperbolic equation]. Vestnik Samarskogo universiteta. Estestvennonauchnaia seriia [Vestnik of Samara University. Natural Science Series], 2018, Vol. 24, no. 4, pp. 7-12. DOI: http://doi.org/10.18287/2541-7525-2018-24-4-7-12 [in Russian].

Статья поступила в редакцию $4 / I X / 2018$.

The article received $4 / I X / 2018$.

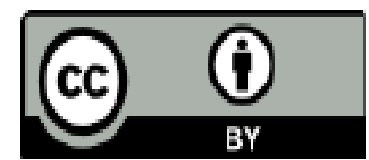

This work is licensed under a Creative Commons Attribution 4.0 International License.

${ }^{2}$ Bogatov Andrey Vladimirovich (andrebogato@mail.ru), Department of Differential Equations and Control Theory, Samara National Research University, 34, Moskovskoye shosse, Samara, 443086, Russian Federation. 